\title{
Testing Gravitational Theories in the Field of the Earth with the SaToR-G Experiment
}

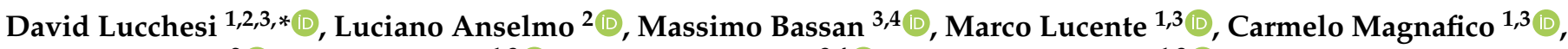 \\ Carmen Pardini ${ }^{2}$ (D), Roberto Peron ${ }^{1,3}\left(\mathbb{D}\right.$, Giuseppe Pucacco ${ }^{3,4}$ (D) and Massimo Visco 1,3 $^{(D)}$ \\ 1 Istituto di Astrofisica e Planetologia Spaziali (IAPS)—Istituto Nazionale di Astrofisica (INAF), \\ Via Fosso del Cavaliere 100, 00133 Roma, Italy; marco.lucente@inaf.it (M.L.); \\ carmelo.magnafico@inaf.it (C.M.); roberto.peron@inaf.it (R.P.); massimo.visco@inaf.it (M.V.) \\ 2 Istituto di Scienza e Tecnologie dell'Informazione (ISTI)—Consiglio Nazionale delle Ricerche, \\ Via G. Moruzzi 1, 56124 Pisa, Italy; luciano.anselmo@isti.cnr.it (L.A.); carmen.pardini@isti.cnr.it (C.P.) \\ 3 Istituto Nazionale di Fisica Nucleare, Sezione di Roma Tor Vergata, Via della Ricerca Scientifica 1, \\ 00133 Roma, Italy; massimo.bassan@roma2.infn.it (M.B.); giuseppe.pucacco@roma2.infn.it (G.P.) \\ 4 Dipartimento di Fisica, Università di Roma Tor Vergata, Via della Ricerca Scientifica 1, 00133 Roma, Italy \\ * Correspondence: david.lucchesi@inaf.it
}

Citation: Lucchesi, D.; Anselmo, L.; Bassan, M.; Lucente, M.; Magnafico, C.; Pardini, C.; Peron, R.; Pucacco, G.; Visco, M. Testing Gravitational Theories in the Field of the Earth with the SaToR-G Experiment. Universe 2021, 7, 192. https://doi.org/ 10.3390/universe7060192

Academic Editors: James A. Isenberg, Gerald B. Cleaver, Lijing Shao, Gonzalo J. Olmo and Giacomo Tommei

Received: 30 April 2021

Accepted: 4 June 2021

Published: 8 June 2021

Publisher's Note: MDPI stays neutral with regard to jurisdictional claims in published maps and institutional affiliations.

Copyright: (c) 2021 by the authors. Licensee MDPI, Basel, Switzerland. This article is an open access article distributed under the terms and conditions of the Creative Commons Attribution (CC BY) license (https:/ / creativecommons.org/licenses/by/ $4.0 /)$.

\begin{abstract}
A new satellite-based experiment in the field of gravitation, SaToR-G, is presented. It aims to compare the predictions of different theories of gravitation in the limit of weak-field and slow-motion. The ultimate goal of SaToR-G is searching for possible "new physics" beyond General Relativity, which represent the state-of-the-art of our current knowledge of gravitational physics. A key role in the above perspective is the theoretical and experimental framework that confines our work. To this end, we will exploit as much as possible the classical framework suggested by R.H. Dicke over fifty years ago.
\end{abstract}

Keywords: general relativity; Dicke's framework; alternative theories of gravitation; weak gravitational fields; PPN formalism; LAGEOS and LARES satellites; orbital residuals

\section{Introduction}

Albert Einstein's theory of General Relativity (GR) [1] is currently the best theory available for interpreting gravitational interaction. The successes of GR are now numerous from the experimental point of view and they confirm this theory as the standard model for gravitation. In addition to today's numerous verifications in the weak field, recent significant results have also been added in other regimes, such as in the case of the detection of gravitational waves and other events that occur in the strong field regime [2-7]. However, the accuracy of the observations is not yet such as to exclude other formulations of gravitation, especially metric theories, as viable alternatives to GR: for example, some observations of gravitational waves concern the constraints on the possible mass of the graviton and the possible polarization of the gravitational waves themselves (two states in GR, up to six states according to other metric theories) [8]. Cosmology is also a field in which many open issues make GR not fully satisfactory in explaining observations, such as the problems related to the horizon, the flatness and the magnetic monopoles in the early and very hot universe, and then inflation, dark matter and dark energy, just to follow a temporal development from the 1960s to the present day [9].

Therefore, alongside the observational/experimental aspects, there are also sufferings linked to the context of the theoretical framework of Einstein's theory that suggest fallacies in the theory, both on large and small scales. This field is indeed not only highly complex but also somewhat thorny, since some of the apparent shortcomings of the theory probably arise just from our limited ability in solving Einstein's field equations. An example is constituted by the rotation curve of the stars at the periphery of a galaxy. Crosta et al. [10] obtained a confirmation of the flatness of the rotation curves of our galaxy without resorting 
to the contribution of the possible presence of dark matter, but including the contribution of gravitomagnetic components (non-diagonal terms of the metric) in a stationary axisymmetric galaxy-scale metric. However, the fact remains that GR, unlike all the other field theories of the Standard Model, does not appear to be quantizable to date, as no one has been able to solve some fundamental problems, such as that of renormalizability [11-13].

The present work is focused on a strategy to compare the predictions of GR with those of other theories of gravitation, both metric and non-metric, in their weak-field and slow-motion limit. While the Parameterized Post-Newtonian (PPN) formalism [14-16] is a standard, very useful tool for such comparisons, we will also try to resume, at least in part, the approach suggested by Dicke more than 50 years ago [17], usually referred to as the Dicke framework. This framework is quite general, it allows to conceive experiments not connected, a priori, with a given physical theory, and it also provides a way to analyze the results of an experiment under primary hypotheses. This activity will be carried out within a new project, SaToR-G, which we present in this paper.

The paper is organized as follows: in Section 2, the main scientific objectives of SaToR$\mathrm{G}$ are briefly summarized. In Section 3, the theoretical and experimental limits within which the studies and measurements of SaToR-G will be confined are fixed. Section 4 is dedicated to recalling the main features of the framework developed by Dicke in 1963. In Section 5, the main results of the previous experiment LARASE are reported. In Section 6, the perspective of SaToR-G are analyzed and deepened on the basis of the results achieved by LARASE. Finally, in Section 7, we outline conclusions and future work.

\section{The Goals of SaToR-G}

SaToR-G (Satellite Tests of Relativistic Gravity) is a new fundamental physics project that aims to test gravitation beyond the predictions of Einstein's GR [1], in search for effects foreseen by alternative theories of gravitation (ATG) [18] and possibly connected with "new physics". SaToR-G is dedicated to measurements of the gravitational interaction in the weak-field and slow-motion (WFSM) limit of GR by means of laser tracking to geodetic passive satellites orbiting the Earth. Indeed, this new program exploits-as quasi-ideal proof masses-the geodynamic laser-ranged satellites LAGEOS [19,20], LAGEOS II [21] and LARES [22] tracked by the powerful Satellite Laser Ranging (SLR) technique [23,24].

The activities of SaToR-G mainly, but not exclusively, focus on metric theories of gravitation, defined in Section 3: GR is obviously the first in this category. In this context, scalar-tensor and vector-tensor theories are of considerable importance. In particular, $f(R)$ theories ${ }^{1}$ are quite interesting to further investigate, such as ATG [25-27]. The main focus of SaToR-G will be twofold: (i) measurement of possible deviations of gravity from the inverse-square law (ISL) for the distance between the Earth and the satellites considered, with possible constraints on a Yukawa-like long-range interaction [28-30] with a typical range $\lambda$ correlated to the semi-major axis of satellites [31-33]; (ii) precise and accurate measurements of some post-Newtonian parameters according to the PPN formalism. These are, in this context, still the most powerful tools for testing the predictions of different theories beyond GR.

The activities of SaToR-G will therefore develop according to two main guidelines: (i) first of all in verifying the predictions of GR in the WFSM limit, and in particular in those areas not yet fully taken into consideration from the experimental point of view, as in the aspects related to the non-linearity of the gravitational interaction; (ii) secondly, in going beyond the predictions of GR in search of possible effects linked to other ATG and, consequently, towards a "new physics".

\section{The Theoretical and Experimental Framework of SaToR-G}

Precisely measuring the orbits of artificial satellites allows to test different metric theories in their most profound aspects related to the curvature of spacetime, to geodesic motion and to the field equations. Metric theories of gravitation share Einstein's Equivalence Principle (EEP) [34], the Lorentzian structure of spacetime and the equations of 
motion. In other words, in all metric theories of gravitation the structure of spacetime is the same, as is the way in which the geometry of spacetime determines the way mass-energy moves in it. What instead profoundly distinguishes GR from the other metric theories of gravitation are the equations of the gravitational field, that is, how the mass-energy of the field orders the geometry of spacetime to curve [18].

As mentioned above, measuring the values of the PPN parameters represents a powerful tool to discriminate among different theories of gravitation. Within the SaToR-G strategy to test theories of gravity, we are also interested in recovering the more general approach from which the PPN formalism itself, in its current version, was basically derived ${ }^{2}$. We will try, as extensively as possible, to test the different theories in the theoretical/experimental framework conceived by Robert Dicke around the mid-1960s [17]. The main idea as the basis of this framework is to generate a set of experiments that is as unbiased as possible considering both classical Newtonian physics and Einstein's GR.

The continuing experimental successes of GR in recent decades made this quest less pressing. On the other hand, during the 1960s and 1970s (and also in the early 1980s), when experimental evidence for the validity of GR was still weak $[35,36]$, several alternative theories were proposed with a certain degree of continuity [37]. In the early 1970s, Kip Thorne and Clifford Will proposed [38] — as a strategy for testing GR - a scheme based on both a Dicke-like approach, as well as an approach based on the nascent, at that time, PPN formalism [39].

However, from a practical point of view, it appears that Dicke's framework was not fully exploited in the past, and the main tests and measurements of GR have actually been based on measurements of the PPN parameters. This aspect is not only largely true in the case of gravitational measurements within the solar system, that is, in the case of weak fields (that Thorne and Will were primarily concerned with, in 1971), but also in the context of almost strong fields such as those tested more recently in relativistic astrophysics [3,6]. It must be said that even in the cases of non-weak fields, the post-Newtonian formalism provides an excellent description of gravitational measurements [40].

For the above reasons, we believe that an effort to reconsider the Dicke framework is appropriate and of interest even in these days, to test the foundation of gravitation, especially in those aspects that are not fully covered by the PPN framework. Below, we briefly outline the basics of Dicke's framework.

\section{The Dicke Framework}

Dicke introduced this conceptual framework in July 1963, within his famous lectures at the Les Houches summer school [17]. Dicke's framework suggests a working hypothesis for analyzing experiments in gravitation, establishing a number of basical mathematical and physical assumptions. This working hypothesis is described in Appendix 4 of Dicke's notes through the articulation of 6 statements that define the limits within which one should move to identify the fundamental characteristics of a relativistic theory of gravitation. The first three points introduced by Dicke focus on the basic mathematical formalism to employ when discussing experiments in gravitation and can be summarized with the following two statements $[18,38]^{3}$ :

1. Spacetime is a 4-dimensional differentiable manifold, with each point in the manifold corresponding to a physical event. The manifold need not a priori have either a metric or an affine connection;

2. The theory of gravity will be expressed in a form that is independent of the particular coordinates used; that is, the equations of gravity and the mathematical entities in them will be put into covariant form.

With two further statements, Dicke introduces two important constraints that an acceptable theory of gravitation should satisfy [18,38]:

1. Gravity must be associated with one or more fields of tensorial character, that is, scalars, vectors, and tensors of various ranks; 
2. The dynamical equations which govern gravity must be derivable from an invariant action principle.

Finally, the last point discussed by Dicke is based on Ockham's razor approach and can be considered a practical guiding principle for choosing the theories of gravitation that are most likely to be correct: Nature likes things as simple as possible [38].

\section{Discussion}

The six pillars highlight the practical/operational aspect that arises from the deep thought of an excellent physicist, a talented experimentalist who was not shy of venturing into theory. In fact, with the above statements and constraints, it seems that Dicke is putting his finger in the sore, or perhaps in the various sores, of Einstein's General Relativity.

The first statement of Dicke's framework is particularly important because it immediately defines the role of a gravitational experiment: it is expected to naturally lead to the existence of a metric and of an affine connection for the differential manifold. The second statement relates to what Einstein's Principle of General Covariance actually is and, therefore, what its validity actually is. So this point goes to the foundations of GR and to those of the ATG. For example, the mentioned covariance is obtained in some cases (as highlighted in [18]), only after the introduction of "absolute" elements or "prior geometric" elements in the theory [41,42]. This means that these elements are not determined by the dynamical equations of the theory and this can be interpreted as a failure of general covariance. This rigidity can be overcome by assuming the invariance of the coordinates instead of their covariance, that is, by a weaker hypothesis.

The two constraints of Dicke's framework seem to strongly confine what an acceptable theory of gravitation is. Indeed, Thorne and Will wrote in this regard [38]: “...These constraints have deep significance; they strongly confine the theory. For this reason, we should be willing to accept them only if they are fundamental to our subsequent arguments. For most applications of the Dicke framework, they are not needed at all. Therefore, we shall usually not assume them. If we ever need and use them, we shall state so explicitly ...".

It is true that, for most applications, these two constraints are not necessary. However, we note that the theories that are still of interest today (see e.g., the state of the art of current ATG discussed in [18]) are those satisfying the two "strong" constraints suggested by Dicke. This was not the case in previous reviews, such as the first edition (1981) of the same book.

As highlighted in Section 3 above, there is a close link between the framework proposed by Dicke and the PPN formalism. In fact, as correctly underlined in [38], two conclusions reached by Dicke within his framework became postulates at the basis of the current PPN formalism: (i) the existence of at least one second rank tensor, the metric $g_{\mu v}$, to be associated with gravity; (ii) the way to describe the response of matter and fields to gravity: $T_{j,}^{\mu v}=0$, where $T_{\mu \nu}$ represents the stress-energy tensor for all matter and non-gravitational fields. In their paper, Thorne and Will also remarked that: " ... It is important for the future that experimenters concentrate not only on measuring the PPN parameters. They should also perform new experiments within the Dicke framework to strengthen - or destroy-the foundation it lays for the PPN framework...".

This is in fact one of the challenges that SaToR-G is called to provide, possibly, an original contribution in the current panorama of experimental physics on the gravitational interaction.

\section{The Legacy from LARASE}

SaToR-G builds on the improved dynamical model of the two LAGEOS and LARES satellites achieved within the previous project LARASE (LAser RAnged Satellites Experiment) [43]. The improvements mainly concern the modeling of the non-conservative forces (NCF) acting on the surface of the three satellites [44-47] and that of the Earth's gravitational field and tides in their precise orbit determination (POD) [47-49]. Regarding the NCF, the main improvements were the development of a model for the spin of the satellites (LASSOS: LArase Satellites Spin mOdel Solutions) and a model for thermal thrust forces (LATOS: LArase Thermal mOdel Solutions). For the gravitational field, the monthly 
solutions of the GRACE mission [50-52] have been implemented in the code used for the POD.

The main results of LARASE in the field of gravitational effects measurements were a precise and accurate measurement of the precession of the argument of pericenter of LAGEOS II [33] and of the Lense-Thirring precession from the analysis of the orbits of the two LAGEOS and LARES satellites $[47,53,54]$. Tables 1 and 2 list the mean orbital elements of the LAGEOS satellites and of LARES, and their main relativistic precessions as predicted by GR respectively.

Table 1. Mean orbital elements of the satellites.

\begin{tabular}{cccccc}
\hline Element & Unit & Symbol & LAGEOS & LAGEOS II & LARES \\
\hline semi-major axis & {$[\mathrm{km}]$} & $a$ & $12,270.00$ & $12,162.07$ & 7820.31 \\
eccentricity & & $e$ & 0.0044 & 0.0138 & 0.0012 \\
inclination & {$[\mathrm{deg}]$} & $i$ & 109.84 & 52.66 & 69.49 \\
\hline
\end{tabular}

Table 2. GR precessions, in millisecond of arc per year (mas/yr), on the right ascension of the ascending node and on the argument of pericenter of LAGEOS, LAGEOS II, and LARES satellites. The LT, dS and Sch labels represent, respectively, the precessions due to the Lense-Thirring, the de Sitter and Schwarzschild effects.

\begin{tabular}{cccc}
\hline Rate in the Element & LAGEOS & LAGEOS II & LARES \\
\hline$\dot{\Omega}_{\mathrm{LT}}$ & +30.67 & +31.50 & +118.48 \\
$\dot{\Omega}_{\mathrm{dS}}$ & +17.64 & +17.64 & +17.64 \\
$\dot{\omega}_{\mathrm{LT}}$ & +31.23 & -57.31 & -334.68 \\
$\dot{\omega}_{\mathrm{Sch}}$ & +3278.77 & +3352.58 & $+10,110.12$ \\
\hline
\end{tabular}

In the first measurement, we have constrained the parameter $\epsilon$, which measures the overall GR precession of the satellite's argument of pericenter (with $\epsilon=1$ in GR and $\epsilon=0$ in Newtonian physics $)^{4}$. We obtained $\epsilon_{\text {meas }}-1=(-0.12 \pm 2.10 \pm 25.4) \times 10^{-3}$, where the first uncertainty represents the error of a non-linear fit to the data-which was estimated from a dedicated sensitivity analysis - while the second uncertainty represents the estimate of the systematic sources of error, mainly dominated by the uncertainty of the Earth's quadrupole coefficient $\bar{C}_{2,0}$ (we refer to [33] for further details).

In the case of the Lense-Thirring precession, the parameter $\mu$, which is used to parameterize the relativistic precession (with $\mu=1$ in GR and $\mu=0$ in Newtonian physics), was measured very accurately with an error budget of about $1.6 \%$ : $\mu_{\text {meas }}-1=$ $(1.5 \pm 7.4 \pm 16) \times 10^{-3}$, where the first uncertainty represents the statistical formal error, at a $95 \%$ confidence level, obtained from a simple linear fit, while the second uncertainty represents the estimate of the systematic sources of error (see [54] for details).

In the case of the first measurement, constraints on non-symmetric and torsional theories of gravitation were set [55-58], leading to an improvement compared to previous results in the literature. Furthermore, a significant result was the constraint on a Yukawa-like interaction with a characteristic range $\lambda$ close to the radius of the Earth and a strength $|\alpha|=|0.5 \pm 8 \pm 101| \times 10^{-12}$ (for the errors, the meaning is the same as in the $\epsilon$ measurement).

\section{Perspectives for SaToR-G}

The two main results of LARASE mentioned in the previous Section 5 can be used to outline a possible route to be followed for future investigations of SaToR-G, in particular in the case of non-metric theories of gravitation. Therefore, we shall consider, in what follows, the two measurements previously introduced, highlighting some of the constraints that can be derived from them, their physical content, and finally the effort needed to go beyond the results achieved by the previous project LARASE. 


\subsection{The Precession of the Orbit Argument of Pericenter}

An example in this direction can be provided by Moffat's non-symmetric theory of gravitation $[55,56]$. In this case the PPN formalism does not apply, since the metric is not symmetric, $g_{\mu \nu} \neq g_{v \mu}$ and, consequently, also the affine connections $\Gamma_{\mu \nu}^{\lambda}$ are not symmetric. From a theoretical point of view, non-symmetric connections imply the existence of other tensor fields, in addition to the metric, which mediate the gravitational interaction. Consequently, we fall within Dicke's framework as a possible natural way to approach the problem of verifying the validity of the proposed theory, that is, whether or not the theory can be considered viable. Furthermore, Moffat's theory provides another useful example, namely a possible link between gravity in the weak-field regime and gravity in strong field, in relation to spacetime singularities ${ }^{5}$. Actually, Ref. [55] highlights how the predictions of this new theory differ significantly from those of GR for compact sources or supermassive stars.

This is indeed a very important aspect from the general point of view of verifying the characteristics of the gravitational interaction. In fact, some ATG predict very slight effects in the limit of weak fields which therefore differ very little from the predictions of GR. Conversely, in the strong field regime, the predictions of these theories may be different from those of GR.

The main characteristic of this non-symmetric theory for gravitation is to associate to a given body $\mathcal{B}$ a charge $\ell_{\mathcal{B}}^{2}$ which arises from the coupling of the non-metric with a vector current $\mathcal{S}^{\mu}$. In a previous study we addressed a parameter $\mathcal{C}_{\mathcal{B}_{1} \mathcal{B}_{2}}$, related to the product of the new charges predicted by the theory and associated with the two gravitationally interacting bodies (in our analysis, the Earth and LAGEOS II): $\mathcal{C}_{\mathcal{B}_{1} \mathcal{B}_{2}}$ is responsible for an additional precession of the argument of pericenter of a satellite. We were able to strictly constrain this parameter: $\mathcal{C}_{\mathcal{B}_{1} \mathcal{B}_{2}}=(0.003 \mathrm{~km})^{4} \pm(0.036 \mathrm{~km})^{4} \pm(0.092 \mathrm{~km})^{4}$ from the constraint in the pericenter of LAGEOS II, see [33] for further details ${ }^{6}$.

One more effect in gravitation, which cannot be interpreted within the PPN formalism, is represented by the possible existence of a new long-range interaction (NLRI). Deviations of the gravitational potential from the Newtonian $1 / r$ law would lead to new weak interactions between macroscopic objects that are predicted by several theories [28-30,60,61]. For these theories, a Yukawa-like parameterization seems general at the lowest order of the interaction and in the non-relativistic limit, independently of the nature of the new field that contributes to mediate the gravitational interaction, that is, of a possible scalar, vector or tensor field (with the exchange of a spin-0, spin-1 or spin-2 light boson, respectively).

Most significantly, these NLRIs may or may not be consistent with EEP. In the second case, non-metric phenomena with tiny, but significant, consequences will be produced in gravitational experiments and also, in particular for our objectives, on the orbits of artificial satellites. The characteristic of these very feeble interactions is the production of deviations for mass separations spanning different orders of magnitude, starting from the sub-millimeter level up to the astronomical scale. The constrain we obtained in [33] for the strength $\alpha$, see previous Section 5, is very stringent and competitive with those obtained - at other distance scales and with other techniques ${ }^{7}$ - for the Moon and for the planets [63-65].

\subsection{The Precession of the Orbit Ascending Node}

The measurement of the Lense-Thirring effect was obtained from the analysis of the precession of the right ascension of the ascending node of the two LAGEOS satellites and of that of LARES [54]. This precession is a consequence of the gravitomagnetic field produced by the rotation of the Earth (i.e., by its angular momentum), which has no analogue in Newton's gravitational theory $[66,67]$. Hereinafter, we first analyze the meaning of the parameter $\mu$, that parameterizes the Lense-Thirring gravitomagnetic precession, and then we analyze some constraints that can be obtained from its measurement.

This parameter, that we have repeatedly measured and constrained with ever increasing precision and accuracy, is not one of the canonical PPN parameters. In fact, it should 
be borne in mind that the PPN formalism does not describe the post-Newtonian limit of any metric theory of gravitation. This aspect, in relation to the Lense-Thirring parameter $\mu$, is clearly explained and proved in [68] (see Chapter 3), where it is explicitly stated: “...the standard ten parameter PPN formalism ... though a very powerful and useful tool to test metric theories, is not sufficient to describe every conceivable metric theory of gravity at the post-Newtonian order ...". Consequently, the ten Newtonian-like potentials underlying the PPN formalism, and therefore also the corresponding ten PPN parameters, are not enough to describe the post-Newtonian approximation of any new and conceivable metric theory of gravitation. In these cases, the more general approach based on Dicke's framework could be a useful tool to integrate the now classic and traditional approach based on the PPN formalism.

The Lense-Thirring precession (or dragging of inertial frames) is often ascribed in the literature to a consequence of the de Sitter effect and consequently described-at the post-Newtonian order-by the PPN parameter $\gamma$ [69-71]. In this case, following the standard PPN gauge, $\mu=(1+\gamma) / 2$ (where $\gamma=1$ in GR) ${ }^{8}$. However, in this case, the gravitomagnetic field associated with the de Sitter effect has nothing to do with the field generated by the motion of mass currents or mass-energy currents relative to other masses, i.e., with the so-called intrinsic gravitomagnetism, as that generated by the intrinsic spin (angular momentum) of a body.

In this case, the gravitomagnetic field holds the exact analogy with a magnetic field $\mathbf{B}$ "seen" in a reference frame moving with a speed $\mathbf{v}$ with respect to an electric charge at rest: $\mathbf{B}=-\left(\mathbf{v} / c^{2}\right) \times \mathbf{E}$, while in the reference frame at rest with the charge there is only an electric field $\mathbf{E}^{9}$. Therefore, this is a gravitomagnetic-like effect that arises by the presence of the gravitoelectric field and the validity of Local Lorentz Invariance (LLI). This gravitomagnetic-like effect can be removed by a Lorentz transformation, and thus it cannot be associated with intrinsic gravitomagnetism, which on the contrary cannot be removed by a Lorentz transformation or a change of coordinates. Therefore, the parameter $\mu$ should be related to intrinsic gravitomagnetism, that is, with the motion of mass currents or mass-energy currents relative to other matter. We refer to [68] for further details.

The right ascension of the ascending node of a satellite is also a key orbital element to constrain the viability of proposed ATG. Among these, torsional ${ }^{10}$ theories $[72,73]$, $f(R)$ theories and in general extended gravity theories [74] can be constrained, even more effectively than with the argument of pericenter, since the expected (and measured) relativistic effects are much smaller in this case, see Table 2. In [73], the authors were able to constrain a linear combination of the two torsion parameters $w_{2}$ and $w_{4}$ in the range $-0.36<w_{2}-w_{4}<+0.44^{11}$. Our recent measurement of the Lense-Thirring effect allows us to further constrain this combination of torsion parameters. Considering our error budget of about $1.6 \%$, we obtain: $w_{2}-w_{4}=-0.006 \pm 0.064$. In the case of an extended gravity theory with $f=f\left(R, R_{\alpha \beta} R^{\alpha \beta}\right)$, the authors of [74] have estimated that with a measurement of $1 \%$ of the Lense-Thirring effect it might be possible to constrain the $m_{Y}$ parameter (one of the effective masses of the theory) to the value $m_{Y}>1.2 \times 10^{-6} \mathrm{~m}^{-1}$. Therefore, with our measurement we can currently set this limit to $m_{Y}>1.9 \times 10^{-6} \mathrm{~m}^{-1}$.

These are just two examples, among many, of how ATG can be constrained by the measurement of the precession of the right ascension of the ascending node of a satellite, regardless of whether these theories are, by their nature, metric.

\subsection{The Role of the Orbit Residuals}

The investigation on a single orbital element when looking for possible effects of a given theory, or of a set of theories, is not entirely satisfactory, since it does not allow us to give a definitive answer on the admissibility of a possible ATG: on the contrary, the possible perturbative effects due to these theories on the entire orbit of the satellites under consideration should be taken into account, in order to highlight their respective signatures. This is the case of the long-term and secular effects, usually those of "easier" interpretation, versus the case of short-term effects, which are more difficult to identify and constrain. 
Prior to these analyses, we will have to establish the goodness of the dynamic model, which is a priori used to model the orbits of the satellites in the data reduction process. This model mainly concerns the reference frames used, the quality of the tracking stations and of the tracking data, and the gravitational and non-gravitational perturbations on the orbit. Once an a priori model has been established, in such a way to be considered sufficiently robust and accurate, a POD of the orbits of the satellites will be performed over an appropriate time interval, in order to obtain the residuals in the different orbital elements of the satellites.

Having decided the arc length of the POD, the state-vector of the satellite at the beginning of each arc is determined and adjusted to best fit the tracking observations. The residuals are obtained by comparing the state-vector of the $n$th arc with the adjusted state-vector of the previous, $(n-1)$ th arc, propagated (with the available models) to the same starting epoch of arc $n$ [76]. The residuals thus computed contain a considerable amount of information. They naturally, and primarily, contain the unmodeled effects that fall within the precision of the POD, but they also contain the unavoidable errors of the models and of the observations. It is important to underline that the residuals thus obtained provide the variation of the orbital elements over the time length of the arc used. Therefore, when divided by the arc length, they directly provide the residuals in the rate of the orbital elements. This is the consequence of the "difference method" described in [76], and has the advantage of relating the temporal variation of the orbital elements with the variation of the perturbative potential with respect to the orbital elements, or with the components of the perturbative acceleration ${ }^{12}$.

In analyzing the residuals obtained from the POD, however, we must ask ourselves whether these can still be corrected for some perturbative effect not yet included in the dynamic model used in the POD. This can occur especially in the case of perturbative effects of non-gravitational origin, that is, in the case of the NCF mentioned in Section 5. The goodness of a new model is therefore based, at this point, on the concordance of its predictions with the signature (or part of it) that characterizes the residuals obtained after the POD. Removing the model predictions, expressed for example through the Gauss equations, from the initial residuals, will provide final residuals suitable to be analyzed to constrain ATG.

\section{Conclusions and Future Works}

We presented the new project—called Satellite Tests of Relativistic Gravity (SaToR-G) which has the final goal of extending the activities of the previous experiment, LARASE, beyond the predictions of Einsten's theory of gravitation. In this regard, a number of activities have been initiated with the aim of performing new kinds of measurements in the field of gravitation with Earth-orbiting laser-ranged satellites. This activity will be based on a theoretical/experimental framework not "simply" described by PPN parameters, but also as adherent as possible to the original framework proposed by R.H. Dicke. After recalling the characteristics of the Dicke framework, we have highlighted a first working strategy that contemplates-as far as possible-these ideas, analyzing the main measurements achieved in LARASE with the constraints already obtained for some alternative theories of gravitation, and also possible constraints obtainable in the future from these (and other) measurements. About this, we have given two new limits. The first concerns possible constraints to a torsional, that is, non-metric, theory of gravitation: $w_{2}-w_{4}=-0.006 \pm 0.064$. The second concerns a metric theory based on the so-called extended gravity: $m_{Y}>1.9 \times 10^{-6} \mathrm{~m}^{-1}$. We believe that these results need to be further analyzed in Dicke's context, beginning with a more in-depth examination of the predicted effects of these theories on the entire orbit of satellites, before an assessment can be given on their reliability.

From a general point of view, we want to stress the importance that the experiments that test the WFSM limit of GR continue to play today, both in the laboratory and in space. Among other things, these experiments guarantee a controlled environment and a 
significant degree of complementarity when experiencing different manifestations of GR. Finally, when discussing Moffat's theory, the difficulty of linking the tests of the various theories of gravitation, starting with GR, made in weak fields with those obtained in strong fields, was very mildly mentioned. These two approaches are so far independent, although complementary: in the first, the static predictions of a theory are verified, while the dynamic ones characterize strong fields. Unfortunately, there is no common framework, to our knowledge, for performing a comparison between the two different regimes. There is evidence that a possible bridge between these regimes can be constituted, under certain conditions, by modern scalar-tensor theories of which Brans-Dicke's is the forerunner. It is indeed a very exciting challenge that should see theoretical physicists as prime movers. In this regard, the Strong Equivalence Principle represents an interesting issue with which to start the study of this very hard problem: indeed, it apparently holds for just one metric theory of gravity, that is, Einstein's geometrodynamics.

Author Contributions: Conceptualization, D.L.; Writing-original draft, D.L.; Writing-review \& editing, D.L., L.A., M.B., M.L., C.M., C.P., R.P., G.P. and M.V. All authors have read and agreed to the published version of the manuscript.

Funding: This research was funded by the National Scientific Committee 2 (CSN2) of the Italian "Istituto Nazionale di Fisica Nucleare" (INFN).

Institutional Review Board Statement: Not applicable.

Informed Consent Statement: Not applicable.

Data Availability Statement: Not applicable.

Acknowledgments: The authors acknowledge the ILRS for providing high-quality laser ranging data of the two LAGEOS satellites and of LARES.

Conflicts of Interest: The authors declare no conflict of interest.

\section{Notes}

1 A class of theories where the the Ricci scalar $R=g^{\mu v} R_{\mu v}$ is replaced, in the action $I$, by a function $f(R)$.

2 The current PPN formalism is based on the works of K. Nordtvedt and C.M. Will previously cited, see Refs. [14-16].

3 Hereinafter we are following Dicke's framework formulation in the words of Thorne and Will [38].

4 Since the bulk of the overall GR precession is the one due to Schwarzschild precession, in the frame of the PPN formalism the parameter $\epsilon$ can be approximated by a combination of the PPN parameter $\beta$ (which describes the nonlinearity of the mass contribution to the metric) and $\gamma$ (which describes space curvature per unit of mass): $\epsilon \simeq(2+2 \gamma-\beta) / 3$.

5 This non-metric theory provides a spherically symmetric static solution that excludes the occurrence of spacetime singularities at $r=0$ in the metric.

6 As highlighted by [56] (see also [59]) if the charge $\ell_{\mathcal{B}}^{2}$ is simply proportional to the mass, we always obtain $\mathcal{C}_{\mathcal{B}_{1} \mathcal{B}_{2}}=0$. Conversely, if the charge scales with the baryon number, we obtain $\mathcal{C}_{\mathcal{B}_{1} \mathcal{B}_{2}} \simeq \ell_{\mathcal{B}}^{4} \xi$, with $\xi \approx 10^{-3}-10^{-2}$.

7 The Lunar Laser Ranging (LLR) and Radar Ranging techniques. In the case of LLR, though the technology of laser tracking is similar to SLR, the orbit and physical model are very different. See for instance [62] for a brief discussion.

8 The complete expression of $\mu$ in this approximation includes an additional contribution equal to $\alpha_{1} / 8$, where $\alpha_{1}$ is a PPN parameter that describes preferred-frame effects and $\alpha_{1}=0$ in GR.

$9 \quad$ We have assumed $v \ll c$.

10 The torsional theories are characterized, like Moffat's theory, by non-symmetric affine connections.

11 This result was based on a previous measurement of the Lense-Thirring effect, see [75], where the error budget of the measurement was estimated to be about $10 \%$ of the relativistic precession.

12 The first case occurs when it is possible to apply the Lagrange equations at the perturbative level, that is when the perturbation corresponds, in the Newtonian sense, to a conservative force. The second case is more general and contemplates the use of Gauss' perturbative equations. 


\section{References}

1. Einstein, A. Die Grundlage der allgemeinen Relativitätstheorie. Ann. Phys. 1916, 354, 769-822. [CrossRef]

2. Kramer, M.; Stairs, I.H.; Manchester, R.N.; McLaughlin, M.A.; Lyne, A.G.; Ferdman, R.D.; Burgay, M.; Lorimer, D.R.; Possenti, A.; D'Amico, N.; et al. Tests of General Relativity from Timing the Double Pulsar. Science 2006, 314, 97-102. [CrossRef] [PubMed]

3. Abbott, B.P.; Abbott, R.; Abbott, T.D.; Abernathy, M.R.; Acernese, F.; Ackley, K.; Adams, C.; Adams, T.; Addesso, P.; Adhikari, R.X.; et al. Observation of Gravitational Waves from a Binary Black Hole Merger. Phys. Rev. Lett. 2016, 116, 061102. [CrossRef] [PubMed]

4. Abbott, B.P.; Abbott, R.; Abbott, T.D.; Abernathy, M.R.; Acernese, F.; Ackley, K.; Araya, M.C.; Barayoga, J.C.; Barish, B.C.; Cerretani, G.; et al. Properties of the binary black hole merger GW150914. Phys. Rev. Lett. 2016, 116, 241102. [CrossRef] [PubMed]

5. Abbott, B.P.; Abbott, R.; Abbott, T.D.; Abernathy, M.R.; Acernese, F.; Ackley, K.; Adams, C.; Adams, T.; Addesso, P.; Adhikari, R.X.; et al. GW150914: Implications for the Stochastic Gravitational-Wave Background from Binary Black Holes. Phys. Rev. Lett. 2016, 116, 131102. [CrossRef]

6. Abbott, B.P.; Abbott, R.; Abbott, T.D.; Acernese, F.; Ackley, K.; Adams, C.; Adams, T.; Addesso, P.; Adhikari, R.X.; Adya, V.B.; et al. Multi-messenger Observations of a Binary Neutron Star Merger. ApJL 2017, 848, L12. [CrossRef]

7. Event Horizon Telescope Collaboration; Akiyama, K.; Alberdi, A.; Alef, W.; Asada, K.; Azulay, R.; Baczko, A.K.; Ball, D.; Baloković, M.; Barrett, J.; et al. First M87 Event Horizon Telescope Results. I. The Shadow of the Supermassive Black Hole. ApJL 2019, 875, L1. [CrossRef]

8. Poisson, E.; Will, C.M. Gravity: Newtonian, Post-Newtonian, Relativistic; Cambridge University Press: Cambridge, UK, 2014; [CrossRef]

9. Debono, I.; Smoot, G.F. General Relativity and Cosmology: Unsolved Questions and Future Directions. Universe 2016, 2, 23. [CrossRef]

10. Crosta, M.; Giammaria, M.; Lattanzi, M.G.; Poggio, E. On testing CDM and geometry-driven Milky Way rotation curve models with Gaia DR2. Mon. Not. R. Astron. Soc. 2020, 496, 2107-2122. [CrossRef]

11. Klauder, J.R. On the meaning of a non-renormalizable theory of gravitation. Gen. Relativ. Gravit. 1975, 6, 13-19. [CrossRef]

12. Goroff, M.H.; Sagnotti, A. The ultraviolet behavior of Einstein gravity. Nucl. Phys. B 1986, 266, 709-736. [CrossRef]

13. Doboszewski, J.; Linnemann, N. How Not to Establish the Non-renormalizability of Gravity. Found. Phys. 2018, 48, 237-252. [CrossRef]

14. Nordtvedt, K. Equivalence Principle for Massive Bodies. II. Theory. Phys. Rev. 1968, 169, 1017-1025. [CrossRef]

15. Will, C.M. Theoretical Frameworks for Testing Relativistic Gravity. II. Parametrized Post-Newtonian Hydrodynamics, and the Nordtvedt Effect. Astrophys. J. 1971, 163, 611-628. [CrossRef]

16. Will, C.M.; Nordtvedt, J.K. Conservation Laws and Preferred Frames in Relativistic Gravity. I. Preferred-Frame Theories and an Extended PPN Formalism. Astrophys. J. 1972, 177, 757-774. [CrossRef]

17. Dicke, R.H. The Theoretical Significance of Experimental Relativity; Blackie and Son Ltd.: London/Glasgow, UK, 1964.

18. Will, C.M. Theory and Experiment in Gravitational Physics; Cambridge University Press: Cambridge, UK, 2018.

19. Johnson, C.W.; Lundquist, C.A.; Zurasky, J.L. (Eds.) The Lageos Satellite. In Proceedings of the Anaheim International Astronautical Federation Congress, Anaheim, CA, USA, 10-16 October 1976.

20. NASA. LAGEOS Phase B Technical Report, NASA Technical Memorandum X-64915; Technical Report TMX-64915; Marshall Space Flight Center, Marshall Space Flight Center: Huntsville, AL, USA, February 1975.

21. Fontana, F. Physical Properties of LAGEOS II Satellite; Technical Report LG-TN-AI-037; Aeritalia: Torino, Itay, 1989.

22. Paolozzi, A.; Ciufolini, I. LARES successfully launched in orbit: Satellite and mission description. Acta Astronaut. 2013, 91,313-321. [CrossRef]

23. Degnan, J.J. Satellite laser ranging: Current status and future prospects. IEEE Trans. Geosci. Remote Sens. 1985, $23,398-413$. [CrossRef]

24. Pearlman, M.R.; Degnan, J.J.; Bosworth, J.M. The International Laser Ranging Service. Adv. Space Res. 2002, 30, 135-143. [CrossRef]

25. Brans, C.; Dicke, R.H. Mach's Principle and a Relativistic Theory of Gravitation. Phys. Rev. 1961, 124, 925-935. [CrossRef]

26. Sotiriou, T.P.; Faraoni, V. f(R) theories of gravity. Rev. Mod. Phys. 2010, 82, 451-497. [CrossRef]

27. De Felice, A.; Tsujikawa, S. f(R) Theories. Living Rev. Relativ. 2010, 13, 3. [CrossRef] [PubMed]

28. Fujii, Y. Dilaton and Possible Non-Newtonian Gravity. Nat. Phys. Sci. 1971, 234, 5-7. [CrossRef]

29. Damour, T.; Piazza, F.; Veneziano, G. Runaway Dilaton and Equivalence Principle Violations. Phys. Rev. Lett. 2002, 89, 081601. [CrossRef]

30. Fischbach, E.; Sudarsky, D.; Szafer, A.; Talmadge, C.; Aronson, S.H. Reanalysis of the Eotvos experiment. Phys. Rev. Lett. 1986, 56, 3-6. [CrossRef] [PubMed]

31. Lucchesi, D.M. The LAGEOS satellites orbit and Yukawa-like interactions. Adv. Space Res. 2011, 47, 1232-1237. [CrossRef]

32. Lucchesi, D.M.; Peron, R. Accurate Measurement in the Field of the Earth of the General-Relativistic Precession of the LAGEOS II Pericenter and New Constraints on Non-Newtonian Gravity. Phys. Rev. Lett. 2010, 105, 231103. [CrossRef] [PubMed]

33. Lucchesi, D.M.; Peron, R. LAGEOS II pericenter general relativistic precession (1993-2005): Error budget and constraints in gravitational physics. Phys. Rev. D 2014, 89, 082002. [CrossRef]

34. Schwartz, H.M. Einstein's comprehensive 1907 essay on relativity, part III. Am. J. Phys. 1977, 45, 899-902. [CrossRef] 
35. Bertotti, B.; Brill, D.; Krotkov, R. Experiments on Gravitation. In Gravitation: An Introduction to Current Research; Wiley \& Sons: New York, NY, USA, 1962.

36. Whitrow, G.J.; Morduch, G.E. Relativistic theories of gravitation: A comparative analysis with particular reference to astronomical tests. Vistas Astron. 1965, 6, 1-67. [CrossRef]

37. Will, C.M. Theory and Experiment in Gravitational Physics; Cambridge University Press: Cambridge, UK, 1993.

38. Thorne, K.S.; Will, C.M. Theoretical Frameworks for Testing Relativistic Gravity. I. Foundations. Astrophys. J. 1971, 163, 595. [CrossRef]

39. Will, C.M. The Confrontation between General Relativity and Experiment. Living Rev. Relativ. 2014, 17, 4. [CrossRef] [PubMed]

40. Will, C.M. Inaugural Article: On the unreasonable effectiveness of the post-Newtonian approximation in gravitational physics. Proc. Natl. Acad. Sci. USA 2011, 108, 5938-5945. [CrossRef]

41. Anderson, J.L. Absolute change in general relativity. In Recent Developments in General Relativity; Pergamon Press: Oxford, UK, $1962 ;$ p. 121.

42. Anderson, J.L. Relativity principles and the role of coordinates in physics. In Gravitation and Relativity; Chiu, H.Y., Hoffmann, W.F., Eds.; Benjamin, Inc.: New York, NY, USA, 1964; Chapter 9.

43. Lucchesi, D.; Anselmo, L.; Bassan, M.; Pardini, C.; Peron, R.; Pucacco, G.; Visco, M. Testing the gravitational interaction in the field of the Earth via satellite laser ranging and the Laser Ranged Satellites Experiment (LARASE). Class. Quantum Grav. 2015, 32, 155012. [CrossRef]

44. Visco, M.; Lucchesi, D.M. Review and critical analysis of mass and moments of inertia of the LAGEOS and LAGEOS II satellites for the LARASE program. Adv. Space Res. 2016, 57, 1928-1938. [CrossRef]

45. Pardini, C.; Anselmo, L.; Lucchesi, D.M.; Peron, R. On the secular decay of the LARES semi-major axis. Acta Astronaut. 2017, 140, 469-477. [CrossRef]

46. Visco, M.; Lucchesi, D.M. Comprehensive model for the spin evolution of the LAGEOS and LARES satellites. Phys. Rev. D 2018, 98, 044034. [CrossRef]

47. Lucchesi, D.M.; Anselmo, L.; Bassan, M.; Magnafico, C.; Pardini, C.; Peron, R.; Pucacco, G.; Visco, M. General Relativity Measurements in the Field of Earth with Laser-Ranged Satellites: State of the Art and Perspectives. Universe $2019,5,141$. [CrossRef]

48. Pucacco, G.; Lucchesi, D.M.; Anselmo, L.; Bassan, M.; Magnafico, C.; Pardini, C.; Peron, R.; Stanga, R.; Visco, M. Earth gravity field modeling and relativistic measurements with laser-ranged satellites and the LARASE research program. In Proceedings of the EGU Conference, Geophysical Research Abstracts, Vienna, Austria, $23-28$ April 2017; Volume 19, EGU2017-13554 .

49. Pucacco, G.; Lucchesi, D.M. Tidal effects on the LAGEOS-LARES satellites and the LARASE program. Celest. Mech. Dyn. Astron. 2018, 130, 66. [CrossRef]

50. Tapley, B.D.; Flechtner, F.; Bettadpur, S.V.; Watkins, M.M. The status and future prospect for GRACE after the first decade. In Proceedings of the Eos Transactions Fall Meeting Supplement Abstract G32A-01, San Francisco, CA, USA, 9-13 December 2013.

51. Cheng, M.; Tapley, B.D.; Ries, J.C. Deceleration in the Earth's oblateness. J. Geophys. Res. Solid Earth 2013, 118, 740-747. [CrossRef]

52. Cheng, M.; Ries, J.C. Decadal variation in Earth's oblateness $\left(\mathrm{J}_{2}\right)$ from satellite laser ranging data. Geophys. J. Int. 2018, 212, 1218-1224. [CrossRef]

53. Lucchesi, D.M.; Visco, M.; Peron, R.; Bassan, M.; Pucacco, G.; Pardini, C.; Anselmo, L.; Magnafico, C. An improved measurement of the Lense-Thirring precession on the orbits of laser-ranged satellites with an accuracy approaching the 1 level. arXiv 2019, arXiv:1910.01941.

54. Lucchesi, D.; Visco, M.; Peron, R.; Bassan, M.; Pucacco, G.; Pardini, C.; Anselmo, L.; Magnafico, C. A 1 Laser-Tracked Satellites. Universe 2020, 6, 139. [CrossRef]

55. Moffat, J.W. New theory of gravitation. Phys. Rev. D 1979, 19, 3554-3558. [CrossRef]

56. Moffat, J.W.; Woolgar, E. Motion of massive bodies: Testing the nonsymmetric gravitation theory. Phys. Rev. D 1988, 37, 918-930. [CrossRef] [PubMed]

57. Hehl, F.W.; von der Heyde, P.; Kerlick, G.D.; Nester, J.M. General relativity with spin and torsion: Foundations and prospects. Rev. Mod. Phys. 1976, 48, 393-416. [CrossRef]

58. Hammond, R.T. Torsion gravity. Rep. Prog. Phys. 2002, 65, 599-649. [CrossRef]

59. Ciufolini, I.; Matzner, R. Non-Riemannian Theories of Gravity and Lunar and Satellite Laser Ranging. Int. J. Mod. Phys. A 1992, 7, 843-852. [CrossRef]

60. Fujii, Y. Scale invariance and gravity of hadrons. Ann. Phys. 1972, 69, 494-521. [CrossRef]

61. Fujii, Y. Scalar-tensor theory of gravitation and spontaneous breakdown of scale invariance. Phys. Rev. D 1974, 9, 874-876. [CrossRef]

62. Nobili, A.M.; Comandi, G.L.; Bramanti, D.; Doravari, S.; Lucchesi, D.M.; Maccarrone, F. Limitations to testing the equivalence principle with satellite laser ranging. Gen. Relativ. Gravit. 2008, 40, 1533-1554. [CrossRef]

63. Williams, J.G.; Turyshev, S.G.; Boggs, D.H. Lunar laser ranging tests of the equivalence principle. Class. Quantum Grav. 2012, 29, 184004. [CrossRef]

64. Standish, E.M.; Newhall, X.X.; Williams, J.G.; Folkner, W.M. JPL Planetary and Lunar Ephemerides, DE403/LE403; Technical Report JPL IOM 314.10-127; Jet Propulsion Laboratory: Pasadena, CA, USA, 1995. 
65. Jurgens, R.F.; Rojas, F.; Slade, M.A.; Standish, E.M.; Chandler, J.F. Mercury Radar Ranging Data from 1987 to 1997. Astron. J. 1998, 116, 486-488. [CrossRef]

66. Thirring, H. Über die Wirkung rotierender ferner Massen in der Einsteinschen Gravitationstheorie. Phys. Z. 1918, 19, 33-39.

67. Lense, J.; Thirring, H. Über den Einfluß der Eigenrotation der Zentralkörper auf die Bewegung der Planeten und Monde nach der Einsteinschen Gravitationstheorie. Phys. Z. 1918, 19, 156-163.

68. Ciufolini, I.; Wheeler, J.A. Gravitation and Inertia; Princeton University Press: Princeton, NJ, USA, 1995.

69. Nordtvedt, K. Existence of the Gravitomagnetic Interaction. Int. J. Theor. Phys. 1988, 27, 1395-1404. [CrossRef]

70. Nordtvedt, K. The Gravitomagnetic Interaction and Its Relationship to Other Relativistic Gravitational Effects. NASA-CR-187736, Final Report. 1991. Available online: https:/ /ntrs.nasa.gov/api/citations/19910012336/downloads/19910012336.pdf (accessed on 30 April 2021).

71. Ashby, N.; Shahid-Saless, B. Geodetic precession or dragging of inertial frames? Phys. Rev. D 1990, 42, 1118-1122. [CrossRef]

72. Mao, Y.; Tegmark, M.; Guth, A.H.; Cabi, S. Constraining torsion with Gravity Probe B. Phys. Rev. D 2007, 76, 104029. [CrossRef]

73. March, R.; Bellettini, G.; Tauraso, R.; Dell'Agnello, S. Constraining spacetime torsion with LAGEOS. Gen. Relativ. Gravit. 2011, 43, 3099-3126. [CrossRef]

74. Capozziello, S.; Lambiase, G.; Sakellariadou, M.; Stabile, A.; Stabile, A. Constraining models of extended gravity using Gravity Probe B and LARES experiments. Phys. Rev. D 2015, 91, 044012. [CrossRef]

75. Ciufolini, I.; Pavlis, E.C. A confirmation of the general relativistic prediction of the Lense-Thirring effect. Nature 2004, 431, 958-960. [CrossRef]

76. Lucchesi, D.M.; Balmino, G. The LAGEOS satellites orbital residuals determination and the Lense Thirring effect measurement. Plan. Space Sci. 2006, 54, 581-593. [CrossRef] 